\title{
Relationship Between Serum Neopterin Level and Peripheral Arterial Plaque in Patients with Type 2 Diabetes
}

\author{
Ren-Hui Wan' \\ Yang Yuan ${ }^{2}$ \\ Wei Hao' \\ Long-Yi Zheng',* \\ Jin Lu $\mathbb{D}^{1, *}$
}

'Department of Endocrinology, Changhai Hospital, Second Military Medical University, Shanghai, People's Republic of China; ${ }^{2}$ Laboratory of Cardiothoracic Surgery Department, Changhai Hospital, Second Military Medical University, Shanghai, People's Republic of China

*These authors contributed equally to this work
Correspondence: Jin Lu

Department of Endocrinology, Changhai Hospital, 168 Changhai Road, Shanghai, 200433, People's Republic of China Tel/FAX +86 21 31161391

Email lujin-sh@139.com
Objective: Neopterin is an inflammatory factor synthesized by monocyte macrophages in response to $\gamma$-interferon. It plays an important role in regulating a variety of physiological and pathological processes, including obesity, diabetes, and cardiovascular disease. This study aims to clarify the relationship between peripheral arterial plaque and serum neopterin in type 2 diabetes.

Methods: We consecutively selected 121 inpatients with type 2 diabetes. After collecting relevant clinical indicators, we collected serum from all patients and measured neopterin levels through enzyme linked immune sorbent assay. Peripheral arterial plaques (the carotid and femoral arteries) were detected by B-mode ultrasound. Multivariate logistic regression was used to determine the independent influencing factors. The correlation between neopterin levels and other variables was analyzed by Spearman correlation analysis. $\mathrm{P}<0.05$ was considered to be statistically significant.

Results: There was no difference in serum neopterin levels between arterial plaques group and no plaques group. Serum neopterin levels in patients with carotid plaque were elevated compared to patients without carotid plaque. Logistic regression analysis showed that a higher serum neopterin level was an independent risk factor for the presence of carotid plaques. Serum neopterin levels were positively correlated with BMI, HOMA-IR, and serum creatinine and negatively correlated with eGFR.

Conclusion: Serum neopterin levels were positively and independently associated with carotid plaque in patients with type 2 diabetes.

Keywords: neopterin, carotid plaques, type 2 diabetes mellitus, atherosclerosis

\section{Introduction}

Along with the development of obesity, sedentary lifestyles, high-energy diets, and population aging, approximately 415 million adults worldwide had diabetes in 2015. ${ }^{1}$ Type 2 diabetes and its complications greatly exacerbate the burden of mortality and disability worldwide. Twenty-seven percentage of diabetics suffer from macrovascular complications, ${ }^{2}$ and macrovascular complications are the main cause of death in type 2 diabetes patients, accounting for $44 \%$ of all-cause mortality. ${ }^{3}$ Diabetes can cause atherosclerosis in all major vascular beds, including coronary, carotid, and lower extremity arteries. ${ }^{4}$ The mechanism of atherosclerosis in diabetes involves oxidative stress and inflammatory response of vascular endothelial cells. ${ }^{5}$ We have confirmed that visfatin serves as a risk factor for atherosclerotic plaques in type 2 diabetes. ${ }^{6}$ Studying the role of different 
inflammatory mediators in peripheral arterial plaques will help clarify the exact pathogenesis of macrovascular complications in type 2 diabetes.

Neopterin is an inflammatory factor synthesized and released by monocyte macrophages in response to $\gamma$ interferon during inflammation. ${ }^{7}$ Multiple studies have verified that neopterin could promote atherosclerosis. The review considers that neopterin is a risk marker for cardiovascular disease, whether it is for unstable angina, acute myocardial infarction, or cardiovascular death. ${ }^{8}$ Increased serum neopterin levels are associated with relapse of acute coronary syndrome and hospitalization of heart failure. ${ }^{9,10}$ In vivo, neopterin may promote plaque growth and apoptosis of vascular smooth muscle cells induced by oxidative stress. ${ }^{11}$ Regarding the relationship between neopterin and diabetes, the serum neopterin levels in patients with diabetes are significantly higher than in healthy and prediabetic people. ${ }^{12,13}$ Serum neopterin appears to be related to impaired insulin secretion and insulin resistance in the development of T2D. ${ }^{14,15}$ Although neopterin has been regarded as a proinflammatory and proatherosclerotic agent, more recent research indicated that neopterin also prevents both vascular inflammation and atherosclerosis and may be induced to counteract the progression of atherosclerotic lesions. ${ }^{16,17}$ Therefore, the correlation between circulating neopterin levels and peripheral plaques in different vascular territories in type 2 diabetes needs to be further investigated.

This study aimed to explore the association between serum neopterin and peripheral arterial plaque in patients with type 2 diabetes and to analyze the difference in neopterin levels in different vascular territories.

\section{Methods}

\section{Study Population}

This cross-sectional study of a single-center cohort was conducted for patients with type 2 diabetes at Changhai Hospital in Shanghai, China. Totally 121 inpatients were consecutively recruited from December 2016 to February 2018. We excluded patients younger than 18 years old, with type 1 diabetes, gestational diabetes, special types of diabetes, acute complications of diabetes (diabetic ketoacidosis and diabetic hyperosmolar coma), acute inflammatory diseases, malignancy, and other endocrine diseases. This study was authorized by the Ethics Committee of Changhai Hospital. The study was conducted in accordance with the Declaration of Helsinki. Written informed consent was obtained before the data collection and analysis.

\section{Clinical and Biochemical Assessment}

The basic information (age, gender, diabetes duration) and anthropometric measurements (height, body weight, waist circumference, hipline circumference) were collected according to standard measurement method. Laboratory data was measured from venous blood and urine samples, which were taken in the morning after an overnight fast of at least $10 \mathrm{~h}$. Blood cell analysis (including white blood cell (WBC) count, total neutrophils, lymphocytes, and platelet count) was measured using the Sysmex XN-9000 analyzer (Sysmex Co., Kobe, Japan). Plasma glucose, serum alanine aminotransferase (ALT), aspartate aminotransferase (AST), $\gamma$-glutamyl transpeptidase (GGT), bilirubin, total bile acid (TBA), blood urea nitrogen (BUN), serum creatinine, uric acid, estimated glomerular filtration rate (eGFR), lipid profile, including total cholesterol, triglycerides, HDL cholesterol (HDL-c), and LDL cholesterol (LDL-c) were performed on HITACHI 7600-120 automatic biochemical analyzer (Hitachi Co., Japan). Hemoglobin A1c (HbAlc) was measured by high-pressure liquid chromatography. Fasting blood glucose (FBG) and postprandial blood glucose (PBG) were measured by the glucose oxidase method. Insulin and C-peptide levels were measured with an automatic analyzer (Roche-E601, Roche Diagnostics, Germany). Urinary albumin-to-creatinine ratio (ACR) was measured using automatic urine microalbumin and creatinine analyzer.

\section{Enzyme-Linked Immunosorbent Assay (ELISA)}

Serum levels of neopterin were measured with commercially available enzyme-linked immunosorbent assay (ELISA) kits according to the manufacturer's instructions (Raybiotech Inc., GA, USA). The intra-assay and interassay coefficients of variation were less than $10 \%$ in enzyme immunoassays.

\section{Peripheral Artery Ultrasound Measurement}

B-mode ultrasound of the carotid and femoral arteries was performed with the Philips rE33 Ultrasound System (Philips Healthcare, Andover, MA). An experienced doctor who was blinded to clinical follow-up data accomplished the analysis of the presence of atheromatous plaques. Arterial plaques were defined as having an 
intima-media thickness (IMT) $\geq 1.3 \mathrm{~mm}$ or a focal protrusion into the lumen with a thickness of at least $50 \%$ more than the adjacent intima-media complex.

\section{Definitions}

The diagnostic criteria for diabetes are strictly based on American Diabetes Association standards. Diabetes duration is calculated from the time when they were first diagnosed with type 2 diabetes. Body mass index (BMI) is defined as body weight $(\mathrm{kg}) /$ height $(\mathrm{m})^{2}$. Waist-hip ratio (WHR) is defined as waist circumference/hipline circumference. HOMA-IR is defined as fasting plasma glucose (mmol/L)* fasting insulin $(\mathrm{mIU} / \mathrm{L}) / 22.5$. eGFR is calculated by the C-MDRD (modified for Chinese) equation: eGFR $=175$ $\mathrm{x}$ (Serum Creatinine) $-1.234 \mathrm{x}$ (Age) $-0.179 \mathrm{x}(0.79$ only if female). ${ }^{18}$

\section{Statistical Analysis}

Using SPSS 22.0 software, categorical data were expressed as proportion. Differences between groups were evaluated by $\chi^{2}$-test. Measurement data of normal distribution were expressed as mean \pm standard deviation (SD), while skewed distribution data were shown as medians (Q1-Q3). Independent-sample $t$ test or MannWhitney $U$-test was used for comparison between groups. Multivariate Logistic regression was used to determine the independent influencing factors. The association between serum neopterin levels and other variables was analyzed using Spearman correlation analysis. P values $<0.05$ were considered to be statistically significant.

The sample size calculations for the comparison between the mean of two independent groups were performed with PASS $11\left(\beta=0.8, \alpha=0.05, \mu_{\beta}=0.84, \mu_{\alpha}=1.96\right.$, $\left.\mathrm{s}_{1}=1.45, \mathrm{~s}_{2}=0.68, \delta=0.68\right)$. The minimal sample size required for each group was 45 subjects.

\section{Results}

\section{General Characteristics of the Study Subjects}

Overall, our study included 121 patients with T2DM who were admitted to our hospital. All patients were then divided into two groups according to the presence or absence of carotid and/or femoral arteries plaques. No plaques in the carotid and/or femoral arteries were found in 41.3\% (50/ 121) of patients. Unexpectedly, as shown in Supplemental

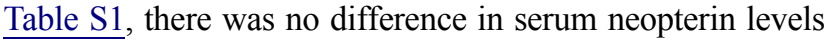

between these two groups. Serum neopterin also cannot serve as a predictor of plaques (Supplemental Table S2).

Comparison of Clinical Indicators and Neopterin Concentrations in Carotid Plaque Group and No Carotid Plaque Group

Considering the different pathogenesis of atherosclerotic plaques in different vascular territories, we further analyzed the relationship between serum neopterin levels with plaques in different arterial territories. All patients were then divided into two groups according to the presence or absence of carotid plaques: carotid plaque group (48 subjects) and no carotid plaque group (73 subjects). As shown in Table 1 and Supplemental Table S3, age was higher in the group with carotid plaques. By contrast, eGFR, serum direct bilirubin, and indirect bilirubin were significantly lower in the group with carotid plaques. Furthermore, serum neopterin levels were elevated in the group with carotid plaques $(\mathrm{P}=0.026)$.

\section{Serum Neopterin as a Predictor of Carotid Plaques}

Multivariable logistic regression analysis was performed in order to assess the independent predictors of the presence of carotid plaques. The above 5 statistically different variables (age, eGFR, serum direct bilirubin, indirect bilirubin, and neopterin) were entered into the full regression model. As demonstrated in Table 2, after controlling for confounding factors, older age, and higher serum neopterin levels were independent risk factors for the presence of carotid plaques. The OR-value of neopterin is 1.689 (1.006, 2.834).

\section{Comparison of Clinical Indicators and Neopterin Concentrations in Femoral Arterial Plaque Group and No Femoral Arterial Plaque Group}

Then, according to the B-mode ultrasonic examination of femoral arteries, they were classified into two groups: femoral arterial plaque group (52 subjects) and no femoral arterial plaque group (69 subjects). As shown in Table 3 and Supplemental Table S4, age and diabetes duration were higher in femoral arterial plaque group. On the other hand, 
Table I Comparison of Carotid Plaque Group and No Carotid Plaque Group

\begin{tabular}{|c|c|c|c|}
\hline Variables & Carotid Plaque $(n=48)$ & No Carotid Plaque $(n=73)$ & P-value \\
\hline Age(years) & $58.63 \pm 9.68$ & $48.55 \pm|3.8|$ & $<0.001$ \\
\hline Gender(male) & $30(62.5 \%)$ & $52(71.2 \%)$ & 0.315 \\
\hline Diabetes duration(years) & $6(1.25-10)$ & $3(I-9)$ & $0.146^{\mathrm{a}}$ \\
\hline $\operatorname{BMI}\left(\mathrm{kg} / \mathrm{m}^{2}\right)$ & $27.89 \pm 3.03$ & $27.77 \pm 4.26$ & 0.861 \\
\hline Waist-to-hip ratio & $0.96 \pm 0.07$ & $0.96 \pm 0.06$ & 0.839 \\
\hline Indirect bilirubin $(\mu \mathrm{mol} / \mathrm{L})$ & $7.5 I \pm 3.12$ & $9.68 \pm 4.42$ & 0.002 \\
\hline Direct bilirubin $(\mu \mathrm{mol} / \mathrm{L})$ & $3.38 \pm 1.4 \mid$ & $4.21 \pm 2.33$ & 0.016 \\
\hline Serum creatinine $(\mu \mathrm{mol} / \mathrm{L})$ & $75.50 \pm 21.75$ & $69.07 \pm 18.63$ & 0.085 \\
\hline $\mathrm{eGFR}(\mathrm{mL} / \mathrm{min})$ & $94.13 \pm 25.32$ & || $0.95 \pm 27.2 \mid$ & 0.001 \\
\hline Total cholesterol(mmol/L) & $4.68 \pm 1.5 I$ & $5.36 \pm 2.21$ & 0.064 \\
\hline Triglycerides $(\mathrm{mmol} / \mathrm{L})$ & $1.72(1.27-2.86)$ & $1.57(1.20-3.09)$ & $0.761^{\mathrm{a}}$ \\
\hline $\mathrm{HDL}$ cholesterol(mmol/L) & $1.00 \pm 0.22$ & $1.09 \pm 0.34$ & 0.070 \\
\hline LDL cholesterol(mmol/L) & $2.75 \pm 1.05$ & $2.87 \pm 1.22$ & 0.565 \\
\hline $\mathrm{ACR}(\mathrm{mg} / \mathrm{g})$ & $17.5(5.25-134.25)$ & $10(5-4 \mid)$ & $0.300^{\mathrm{a}}$ \\
\hline Fasting plasma glucose $(\mathrm{mmol} / \mathrm{L})$ & $9.19 \pm 2.90$ & $9.47 \pm 3.22$ & 0.630 \\
\hline Ih postprandial plasma glucose (mmol/L) & $15.90 \pm 4.08$ & $16.10 \pm 3.89$ & 0.782 \\
\hline $2 \mathrm{~h}$ postprandial plasma glucose $(\mathrm{mmol} / \mathrm{L})$ & $|8.23 \pm 4.0|$ & $17.86 \pm 4.50$ & 0.646 \\
\hline Fasting insulin(mIU/L) & |3.| (6.85-|8.95) & II.4(7.05-17.0) & $0.433^{\mathrm{a}}$ \\
\hline Fasting C-peptide( $\mu \mathrm{g} / \mathrm{L})$ & $2.37(1.82-3.34)$ & $2.50(1.56-3.13)$ & $0.649^{\mathrm{a}}$ \\
\hline Ih postprandial insulin(mIU/L) & $25.3(16.05-56.95)$ & $28.0(13.15-45.2)$ & $0.578^{\mathrm{a}}$ \\
\hline Ih postprandial C-peptide( $\mu g / L)$ & $3.62(2.72-5.96)$ & $3.35(2.36-4.97)$ & $0.395^{\mathrm{a}}$ \\
\hline $2 \mathrm{~h}$ postprandial insulin(mIU/L) & $31.25(13.68-56.28)$ & $29.3(15.35-56.35)$ & $0.80 \mathrm{I}^{\mathrm{a}}$ \\
\hline 2 h postprandial C-peptide $(\mu \mathrm{g} / \mathrm{L})$ & $4.59(3.38-7.17)$ & $4.78(3.04-6.53)$ & $0.494^{\mathrm{a}}$ \\
\hline HOMA-IR & $4.68(3.25-8.33)$ & $4.62(3.00-8.06)$ & $0.468^{\mathrm{a}}$ \\
\hline Hemoglobin Alc(\%) & $9.61 \pm 1.89$ & $9.53 \pm 2.18$ & 0.852 \\
\hline Neopetrin(ng/mL) & $2.02(1.57-2.89)$ & $1.80(1.48-2.26)$ & $0.026^{\mathrm{a}}$ \\
\hline
\end{tabular}

Notes: ${ }^{\mathrm{a}}$ compared by the Mann-Whitney test.

Abbreviations: BMI, body mass index; eGFR, estimated glomerular filtration rate; HDL cholesterol, high-density lipoprotein cholesterol; LDL cholesterol, low-density lipoprotein cholesterol; ACR, urinary albumin-to-creatinine ratio.

Table 2 Independent Factors for Carotid Plaques by Correlations Between Serum Neopterin Multivariable Logistic Regression Analysis

\begin{tabular}{|l|c|c|}
\hline Parameters & OR (95\% Cl) & P-value \\
\hline Age & $1.06 I(I .020,1.104)$ & 0.003 \\
Direct bilirubin & $0.853(0.578, I .258)$ & 0.422 \\
Indirect bilirubin & $0.908(0.758, I .087)$ & 0.293 \\
eGFR & $0.990(0.973,1.007)$ & 0.246 \\
Neopterin & $1.689(I .006,2.834)$ & 0.047 \\
\hline
\end{tabular}

Abbreviations: OR, odds ratio; $\mathrm{Cl}$, confidence interval; eGFR, estimated glomerular filtration rate.

serum indirect bilirubin, alanine aminotransferase, aspartate aminotransferase, $\gamma$-Glutamyl transpeptidase, and total cholesterol were significantly lower in femoral arterial plaque group. There was no statistical difference in serum neopterin levels between the two groups $(\mathrm{P}=0.130$ ). Multivariable logistic regression analysis revealed that serum neopterin levels were not independent risk factors for the presence of femoral plaques (Supplemental Table S5).

\section{Levels and Clinical Indicators}

In order to find out the relevant factors that affect the serum neopterin levels in patients with type 2 diabetes, the correlation between the serum neopterin levels and other clinical parameters was analyzed by Spearman correlation analysis. As shown in Table 4 and Supplemental Table S6, serum neopterin levels were positively correlated with BMI $\left(r_{s}=0.191, P=0.036\right)$, serum creatinine $\left(r_{s}\right.$ $=0.327, \mathrm{P}<0.001)$, HOMA-IR $\left(\mathrm{r}_{\mathrm{s}}=0.184, \mathrm{P}=0.043\right)$, and negatively correlated with eGFR $\left(r_{s}=0.358, \mathrm{P}<0.001\right)$.

\section{Discussion}

Neopterin is an inflammatory factor secreted by monocyte macrophages. $^{7}$ In the past two decades, neopterin has received more and more attention in the pathogenesis of cardiovascular complications. ${ }^{19-21}$ In diabetic patients, it is also confirmed that neopterin is an independent predictor for fatal ischemic heart disease. ${ }^{22}$ 
Table 3 The Comparison of Femoral Plaque Group and No Femoral Plaque Group

\begin{tabular}{|c|c|c|c|}
\hline Variables & Femoral Plaque $(n=52)$ & No Femoral Plaque $(n=69)$ & P-value \\
\hline Age(years) & $58.31 \pm 10.38$ & $48.20 \pm 13.58$ & $<0.001$ \\
\hline Gender(male) & $38(73.1 \%)$ & $44(63.8 \%)$ & 0.278 \\
\hline Diabetes duration(years) & $6(2-11.75)$ & $3(0.54-8)$ & $0.027^{\mathrm{a}}$ \\
\hline $\operatorname{BMI}\left(\mathrm{kg} / \mathrm{m}^{2}\right)$ & $27.36 \pm 2.79$ & $28 .|6 \pm 4.4|$ & 0.228 \\
\hline Waist-to-hip ratio & $0.96 \pm 0.06$ & $0.96 \pm 0.07$ & 0.941 \\
\hline Indirect bilirubin $(\mu \mathrm{mol} / \mathrm{L})$ & $7.92 \pm 2.93$ & $9.49 \pm 4.68$ & 0.026 \\
\hline Direct bilirubin $(\mu \mathrm{mol} / \mathrm{L})$ & $3.5 I \pm 1.17$ & $4.17 \pm 2.49$ & 0.058 \\
\hline Serum creatinine $(\mu \mathrm{mol} / \mathrm{L})$ & $74.29 \pm 21.62$ & $69.61 \pm 18.76$ & 0.206 \\
\hline eGFR(mL/min) & $98.99 \pm 26.58$ & $108.26 \pm 27.93$ & 0.068 \\
\hline Total cholesterol $(\mathrm{mmol} / \mathrm{L})$ & $4.56 \pm 1.47$ & $5.49 \pm 2.23$ & 0.010 \\
\hline Triglycerides(mmol/L) & $1.62(1.15-2.86)$ & $1.77(1.26-3.35)$ & $0.316^{\mathrm{a}}$ \\
\hline HDL cholesterol(mmol/L) & $1.03 \pm 0.33$ & $1.08 \pm 0.28$ & 0.435 \\
\hline LDL cholesterol(mmol/L) & $2.61 \pm 1.10$ & $2.98 \pm 1.17$ & 0.084 \\
\hline $\mathrm{ACR}(\mathrm{mg} / \mathrm{g})$ & $12(4.25-126.75)$ & II (5-45.57) & $0.755^{\mathrm{a}}$ \\
\hline Fasting plasma glucose $(\mathrm{mmol} / \mathrm{L})$ & $8.91 \pm 2.49$ & $9.70 \pm 3.45$ & 0.165 \\
\hline Ih postprandial plasma glucose(mmol/L) & $15.96 \pm 3.75$ & $16.07 \pm 4.12$ & 0.889 \\
\hline $2 \mathrm{~h}$ postprandial plasma glucose(mmol/L) & $18.14 \pm 3.85$ & $|7.9| \pm 4.64$ & 0.777 \\
\hline Fasting insulin(mIU/L) & |2.I(6.78-18.95) & $12.2(7.4-17.6)$ & $0.900^{\mathrm{a}}$ \\
\hline Fasting C-peptide( $\mu g / L)$ & $2.47(1.77-3.14)$ & $2.4 \mathrm{I}(\mathrm{I} .6 \mathrm{I}-3.20)$ & $0.992^{\mathrm{a}}$ \\
\hline Ih postprandial insulin(mlU/L) & $28.7(19.0-63.08)$ & $23.8(I 1.85-45.2)$ & $0.079^{\mathrm{a}}$ \\
\hline Ih postprandial C-peptide( $\mu \mathrm{g} / \mathrm{L})$ & $3.67(2.44-5.80)$ & $3.32(2.38-4.91)$ & $0.250^{\mathrm{a}}$ \\
\hline 2 h postprandial insulin(mlU/L) & $30.95(|7.55-6| .93)$ & $26.4(13.2-50.15)$ & $0.124^{\mathrm{a}}$ \\
\hline 2 h postprandial C-peptide $(\mu g / L)$ & $4.75(3.33-7.13)$ & $4.56(2.97-6.53)$ & $0.308^{\mathrm{a}}$ \\
\hline HOMA-IR & $4.55(2.92-7.98)$ & $4.80(3.10-8.83)$ & $0.755^{\mathrm{a}}$ \\
\hline Hemoglobin AIc(\%) & $9.32 \pm 1.86$ & $9.75 \pm 2.20$ & 0.261 \\
\hline Neopetrin(ng/mL) & $2.00(\mid .56-2.7 I)$ & $\mathrm{I} .80(\mid .5 \mathrm{I}-2.28)$ & $0.130^{\mathrm{a}}$ \\
\hline
\end{tabular}

Notes: a compared by the Mann-Whitney test.

Abbreviations: BMI, body mass index; eGFR, estimated glomerular filtration rate; HDL cholesterol, high-density lipoprotein cholesterol; LDL cholesterol, low-density lipoprotein cholesterol; ACR, urinary albumin-to-creatinine ratio.

Diabetes mellitus is a major risk factor for peripheral artery disease. In this study, we tried to establish an association between peripheral arterial plaques, as detected by ultrasonography, in two different territories, including the carotid and femoral arteries, and the serum neopterin levels. According to our results, serum neopterin levels in type 2 diabetes patients with carotid plaque were significantly higher than group without carotid plaque. Interestingly, there was no statistical difference in the

Table 4 Correlation of Neopterin Levels and Clinical Variables

\begin{tabular}{|l|c|c|}
\hline \multirow{2}{*}{ Parameters } & \multicolumn{2}{|c|}{ Neopterin } \\
\cline { 2 - 3 } & $\mathbf{r}_{\mathbf{s}}$ & P-value \\
\hline BMI & 0.191 & 0.036 \\
Serum creatinine & 0.327 & $<0.001$ \\
eGFR & -0.358 & $<0.001$ \\
HOMA-IR & 0.184 & 0.043 \\
\hline
\end{tabular}

Abbreviations: BMI, body mass index; eGFR, estimated glomerular filtration rate. levels of neopterin between femoral arterial plaque group and no femoral arterial plaque group. The difference between the carotid arterial plaque group and the femoral arterial plaque group may attribute to the plaque composition. The area and number of macrophages in carotid arterial plaque are higher than those in femoral arterial plaque, ${ }^{23}$ while neopterin is secreted by activated monocyte macrophages. Except for macrophages, the distribution of other inflammatory molecular markers is different in carotid and femoral atherosclerotic plaques. ${ }^{24}$ Regional differences in the hemodynamic profile prime the endothelial phenotype to respond distinctly to such systemic risk factors as hypercholesterolemia, genetics, immune status, and oxidative stress. ${ }^{25}$ So neopterin may play a unique role in the process of carotid atherosclerosis, which is different from other vascular territory.

Briefly, after controlling for other confounding factors, neopterin was still an independent predictor for carotid plaque in patients with type 2 diabetes. Migration of 
monocyte macrophages through the endothelium represents a key step of atherosclerosis, and the relationship between neopterin and atherosclerosis could be partly explained by the associated activation of macrophages. Oxidative stress seems to play a vital role, ${ }^{26}$ because neopterin amplifies the activation of reactive oxygen species mediated cellular processes. ${ }^{27}$ De Rosa et al held that activated monocyte macrophages in local atherosclerotic areas obviously increase neopterin levels, thus inducing the expression of adhesion molecules in endothelial cells. These adhesion molecules result in increased binding of lymphocytes, platelets, and other monocytes. Subsequent endothelial activation causes expression of tissue factor, finally leading to the occurrence of atherosclerosis. ${ }^{28}$ Nevertheless, the specific molecular mechanisms involved in carotid arterial plaques need to be further studied.

Neopterin is excreted through the kidneys in vivo. ${ }^{29}$ We also found that serum neopterin levels were positively correlated with serum creatinine, and negatively correlated with eGFR in patients with type 2 diabetes. Similarly, a prospective cohort study confirmed that the serum creatinine of high-level neopterin increased faster than low-level neopterin group in diabetic nephropathy patients. $^{30}$ Spearman correlation analysis showed positive correlations between neopterin levels and BMI as well. Neopterin levels in obese people were higher than in non-obese people, ${ }^{31}$ and the level of neopterin decreased after weight loss. ${ }^{32}$ Likewise, subjects with WHR $>0.9$ had higher serum neopterin levels. ${ }^{33}$ The association between neopterin and BMI may ascribe to chronic low-grade inflammation in obese people. ${ }^{34}$ In this study, we revealed that serum neopterin levels were positively correlated with HOMA-IR, which is consistent with the previous report performed in newly diagnosed patients with T2D who did not take any hypoglycemic agents. $^{14}$

In addition, there are several limitations to be discussed. First of all, our study was based on crosssectional data with a small sample size from a single center. Prospective studies with larger samples and multiple centers are needed to explore the role of serum neopterin in carotid and lower extremity arterial plaques of type 2 diabetes. Secondly, we did not collect the history of drug therapy, such as insulin, oral hypoglycemic drugs, and other factors which may have impact on atherosclerosis. Finally, atherosclerosis has a complicated pathological process, and it involves many molecular markers. Only one biomarker is not enough to view the whole picture of atherosclerotic plaque formation.

\section{Conclusions}

We underline that serum neopterin levels were positively and independently associated with carotid plaque in patients with type 2 diabetes. These results also imply that neopterin could be a new therapeutic target both for the prevention and the treatment of carotid plaque.

\section{Abbreviations}

BMI, body mass index; eGFR, estimated glomerular filtration rate; HDL cholesterol, high-density lipoprotein cholesterol; LDL cholesterol, low-density lipoprotein cholesterol; ACR, urinary albumin-to-creatinine ratio.

\section{Data Sharing Statement}

The datasets used and/or analyzed during the current study are available from the corresponding author on reasonable request.

\section{Ethics Approval and Consent to Participate}

The study was approved by the ethics committee of the Changhai Hospital, Clinical Research Ethics Committee. Written informed consent was obtained before the data collection and analysis.

\section{Author Contributions}

All authors made substantial contributions to conception and design, acquisition of data, or analysis and interpretation of data; took part in drafting the article or revising it critically for important intellectual content; agreed to submit to the current journal; gave final approval for the version to be published; and agree to be accountable for all aspects of the work.

\section{Funding}

This research was supported by research project on aging, maternal, and child health from Shanghai Health Commission (2020YJZX0131), medical innovation research project from Shanghai Science and Technology Committee (20Y11905200) and the project from National Natural Science Foundation of China (81672831).

\section{Disclosure}

The authors report no conflicts of interest in this work. 


\section{References}

1. Zheng Y, Ley SH, Hu FB. Global aetiology and epidemiology of type 2 diabetes mellitus and its complications. Nat Rev Endocrinol. 2018;14(2):88-98. doi:10.1038/nrendo.2017.151

2. Litwak L, Goh SY, Hussein Z, Malek R, Prusty V, Khamseh ME. Prevalence of diabetes complications in people with type 2 diabetes mellitus and its association with baseline characteristics in the multinational A1chieve study. Diabetol Metab Syndr. 2013;5(1):57. doi:10.1186/1758-5996-5-57

3. Marso SP, Hiatt WR. Peripheral arterial disease in patients with diabetes. J Am Coll Cardiol. 2006;47(5):921-929. doi:10.1016/j. jacc.2005.09.065

4. Forbang NI, McDermott MM, Liao Y, et al. Associations of diabetes mellitus and other cardiovascular disease risk factors with decline in the ankle-brachial index. Vasc Med. 2014;19(6):465-472. doi: $10.1177 / 1358863 X 14554033$

5. Paneni F, Beckman JA, Creager MA, Cosentino F. Diabetes and vascular disease: pathophysiology, clinical consequences, and medical therapy: part I. Eur Heart J. 2013;34(31):2436-2443. doi:10.1093/eurheartj/eht149

6. Zheng L-Y, Xu X, Wan R-H, Xia S, Huang Q. Association between serum visfatin levels and atherosclerotic plaque in patients with type 2 diabetes. Diabetol Metab Syndr. 2019;11(1):60. doi:10.1186/ s13098-019-0455-5

7. Steven G, Gregory BP, Angus L. Neopterin, Inflammation, and Oxidative Stress: what Could We Be Missing? Antioxidants. 2018;7 (7):80. doi:10.3390/antiox7070080

8. Sugioka K, Naruko T, Matsumura Y, et al. Neopterin and atherosclerotic plaque instability in coronary and carotid arteries. J Atheroscler Thromb. 2010;17(11):1115-1121. doi:10.5551/jat.4606

9. Nazer B, Ray KK, Sloan S, et al. Prognostic utility of neopterin and risk of heart failure hospitalization after an acute coronary syndrome. Eur Heart J. 2011;32(11):1390-1397. doi:10.1093/eurheartj/ehr032

10. Ray KK, Morrow DA, Sabatine MS, et al. Long-term prognostic value of neopterin: a novel marker of monocyte activation in patients with acute coronary syndrome. Circulation. 2007;115 (24):3071-3078. doi:10.1161/CIRCULATIONAHA.106.666511

11. Gieseg SP, Crone EM, Flavall EA, Amit Z. Potential to inhibit growth of atherosclerotic plaque development through modulation of macrophage neopterin/7,8-dihydroneopterin synthesis. $\mathrm{Br}$ $J$ Pharmacol. 2008;153(4):627-635. doi:10.1038/sj.bjp.0707408

12. Grossmann V, Schmitt VH, Zeller T, et al. Profile of the Immune and Inflammatory Response in Individuals With Prediabetes and Type 2 Diabetes. Diabetes Care. 2015;38(7):1356-1364. doi:10.2337/dc14-3008

13. Unuvar S, Tanriverdi Z, Aslanhan H. Potential Prognostic Role of Immune System Activation Marker Neopterin in Patients with Type 2 Diabetes. J Med Biochem. 2018;37(4):465-469. doi:10.2478/jomb2018-0004

14. Lee JE, Oh TJ, Moon JH, Park KS, Jang HC, Choi SH. Serum Neopterin Concentration and Impaired Glucose Metabolism: relationship With beta-Cell Function and Insulin Resistance. Front Endocrinol (Lausanne). 2019;10:43. doi:10.3389/fendo.2019.00043

15. Cuta A, Drugan C, Roman G, Rusu A, Cătană CS. Evaluation of Chitotriosidase and Neopterin as Biomarkers of Microvascular Complications in Patients with Type 1 Diabetes Mellitus. Diagnostics. 2021;11(2):263. doi:10.3390/diagnostics11020263

16. Lyu Y, Jiang X, Dai W. The roles of a novel inflammatory neopterin in subjects with coronary atherosclerotic heart disease. Int Immunopharmacol. 2015;24(2):169-172. doi:10.1016/j. intimp.2014.11.013

17. Fredericks S. Neopterin Counters Vascular Inflammation and Atherosclerosis. J Am Heart Assoc. 2018.
18. Ma Y-C, Zuo L, Chen J-H. Modified Glomerular Filtration Rate Estimating Equation for Chinese Patients with Chronic Kidney Disease. J Am Soc Nephrol. 2006;17(10):2937. doi:10.1681/ ASN.2006040368

19. Auer J, Berent R, Lassnig E, Weber T, Eber B. Prognostic significance of immune activation after acute coronary syndromes. $\mathrm{J}$ Am Coll Cardiol. 2002;39(11):1878. doi:10.1016/S0735-1097(02)01875-2

20. Zouridakis E, Avanzas P, Arroyo-Espliguero R, Fredericks S, Kaski JC. Markers of inflammation and rapid coronary artery disease progression in patients with stable angina pectoris. Circulation. 2004;110(13):1747-1753. doi:10.1161/01.CIR.0000142664.18739.92

21. Avanzas P, Arroyo-Espliguero R, Quiles J, Roy D, Kaski JC. Elevated serum neopterin predicts future adverse cardiac events in patients with chronic stable angina pectoris. Eur Heart J. 2005;26 (5):457-463. doi:10.1093/eurheartj/ehi111

22. Vengen IT, Dale AC, Wiseth R, Midthjell K, Videm V. Neopterin predicts the risk for fatal ischemic heart disease in type 2 diabetes mellitus: long-term follow-up of the HUNT 1 study. Atherosclerosis. 2009;207(1):239-244. doi:10.1016/j.atherosclerosis.2009.04.003

23. Herisson F, Heymann M, Chétiveaux M, et al. Carotid and femoral atherosclerotic plaques show different morphology. Atherosclerosis. 2011;216(2):348-354. doi:10.1016/j.atherosclerosis.2011.02.004

24. Zhou W, Chai H, Ding R, Lam H. Distribution of inflammatory mediators in carotid and femoral plaques. $J$ Am Coll Surg. 2010;211(1):92-98. doi:10.1016/j.jamcollsurg.2010.02.054

25. VanderLaan P, Reardon C, Getz G. Site specificity of atherosclerosis: site-selective responses to atherosclerotic modulators. Arterioscler Thromb Vasc Biol. 2004;24(1):12-22. doi:10.1161/01. ATV.0000105054.43931.f0

26. Oettl K, Reibnegger G. Pteridine derivatives as modulators of oxidative stress. Curr Drug Metab. 2002;3(2):203-209. doi:10.2174/ 1389200024605127

27. Hoffmann G, Wirleitner B, Fuchs D. Potential role of immune system activation-associated production of neopterin derivatives in humans. Inflammation Researc. 2003;52(8):313-321. doi:10.1007/s00011003-1181-9

28. De Rosa S, Cirillo P, Pacileo M, et al. Neopterin: from forgotten biomarker to leading actor in cardiovascular pathophysiology. Curr Vasc Pharmacol. 2011;9(2):188-199. doi:10.2174/ 157016111794519372

29. Sucher R, Schroecksnadel K, Weiss G, Margreiter R, Fuchs D, Brandacher G. Neopterin, a prognostic marker in human malignancies. Cancer Lett. 2010;287(1):13-22. doi:10.1016/j. canlet.2009.05.008

30. Weiss M, Rodby R, Justice A, Hricik D. Free pentosidine and neopterin as markers of progression rate in diabetic nephropathy. Collaborative Study Group. Kidney Int. 1998;54(1):193-202. doi:10.1046/j.1523-1755.1998.00982.x

31. Abdou A, Magour G, Mahmoud M. Evaluation of some markers of subclinical atherosclerosis in Egyptian young adult males with abdominal obesity. Br $\quad J$ Biomed Sci. 2009;66(3):143-147. doi:10.1080/09674845.2009.11730261

32. Aasbrenn M, Farup PG, Videm V. Changes in C-reactive protein, neopterin and lactoferrin differ after conservative and surgical weight loss in individuals with morbid obesity. Sci Rep. 2019;9(1):17695. doi:10.1038/s41598-019-54107-z

33. Bozdemir AE, Barutcuoglu B, Dereli D, Kabaroglu C, Habif S, Bayindir O. C-reactive protein and neopterin levels in healthy non-obese adults. Clin Chem Lab Med. 2006;44(3):317-321. doi:10.1515/CCLM.2006.055

34. Cox AJ, West NP, Cripps AW. Obesity, inflammation, and the gut microbiota. Lancet Diabetes Endocrinol. 2015;3(3):207-215. doi:10.1016/S2213-8587(14)70134-2 


\section{Publish your work in this journal}

Diabetes, Metabolic Syndrome and Obesity: Targets and Therapy is an international, peer-reviewed open-access journal committed to the rapid publication of the latest laboratory and clinical findings in the fields of diabetes, metabolic syndrome and obesity research. Original research, review, case reports, hypothesis formation, expert opinion and commentaries are all considered for publication. The manuscript management system is completely online and includes a very quick and fair peer-review system, which is all easy to use. Visit http://www.dovepress.com/testimonials.php to read real quotes from published authors. 